\title{
Magnetoelectric effect in a Terfenol-D and electret capacitor system
}

\author{
Tian-Xiang Gao, Yan-Wei Zhou, Su-Su Wang, Yun Zhou \\ College of Science, China Jiliang University, Hangzhou 310018, China
}

\begin{abstract}
A capacitor system based on a Terfenol-D rod and an electret film has been proposed. We used the traditional dynamic measurement to investigate the magnetoelectric effect of the system at the frequency of $50 \mathrm{kHz}$. The results show that the ME coefficient of the system is significantly affected by the DC bias magnetic field. The maximum tunability of the magnetoelectric effect based on the DC bias magnetic field is more than $10 \%$. The induced AC voltage of effective values increases almost lineally with increasing AC signal magnetic field. The detected magnetoelectric effect of the system exhibits hysteresis, and the magnetoelectric hysteresis loops are almost unchanged with the test period. These findings provide a type of capacitor for potential applications in magnetic detections and magnetoelectric sensors.
\end{abstract}

\section{Introduction}

In previous decades, materials with magnetoelectric (ME) effect have drawn significant interest for their wide applicability in ME multifunctional devices [1-3]. Because the ME effect of single-phase ME material is weak, many researches focus on complex composites and combined configurations [4-5]. In these composites and configurations, most of them are made of magnetostrictive materials and piezoelectric materials [67]. Electrets have the property of possessing a permanent electric field at their surface [8], which make them be widely used in energy harvesters, air filters, microphones, and so on [9-11]. However, Electrets-based ME configurations are seldom reported.

Taking the above-mentioned factors into consideration, the aim of this study is to investigate the ME effect of a capacitor system based on magnetostrictive materials and electrets. In this system, the magnetostrictive material can stretch out and draw back under an applied magnetic field, which change the distance between the two plates of the capacitor. Then an output voltage between the two plates can be measured. Thus a ME response is observed. This ME response of this capacitor system is quite different from that of ME materials. Therefore, it is necessary to investigate the ME effect of this system, which also can provide useful information for practical applications of electrets.

\section{Experimental details}

Figure 1 illustrates the geometry and working principle of the capacitor system. The system consists of a magnetostrictive Terfenol-D rod, a dielectric elastic latex plate, an electret film plate, and a copper plate as shown in Figure 1a. Both the electret film and the Terfenol-D rod were commercially obtained with the same diameter of $8 \mathrm{~mm}$. The length of the Terfenol-D rod is about 30 $\mathrm{mm}$, and the thicknesses of the latex pate and electret film are approximately $0.15 \mathrm{~mm}$ and $0.02 \mathrm{~mm}$, respectively. The electret film was attached to the copper plate using conductive glue. The saturation magnetostriction of Terfenol-D, the surface charge density of the electret film, and the relative dielectric constant of the latex plate were reported to be approximately $1300 \mathrm{ppm}, 62 \mu \mathrm{C} / \mathrm{m}^{2}$, and 8 respectively [12]. As can be seen in Figure 1a, the Terfenol-D and copper plate were very closely spaced-filled with the latex plate and electret film, and faced one another. The Terfenol-D, latex pate, electret film and copper plate formed a parallel plate capacitor system.

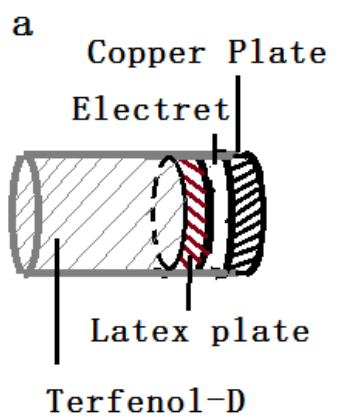

b

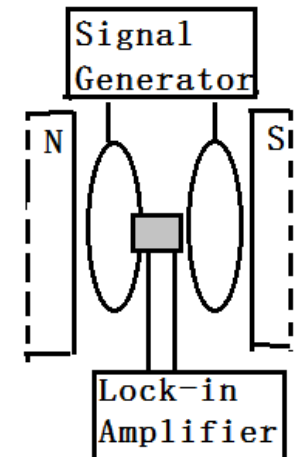

Figure 1. (a) Schematic of the system and (b) the testing setup used for ME effect measurement. The Helmholtz coils was connected to a signal generator with a power amplifier and the output voltage from the system was measured by lock-in amplifier.

\footnotetext{
a Corresponding author: zhouyun@cjlu.edu.cn
} 
The system was placed in a plastic capsule and two leads from the capacitor were connected to a lock-in amplifier as mentioned before [12]. The ME effect measurement was performed using the dynamic method, as shown in Figure 1b. The ME coefficient $(\alpha)$ was determined by measuring the output voltage under a small applied sinusoidal AC magnetic field of $50 \mathrm{kHz}$ (generated by Helmholtz coils, 0.45 Oe) superimposed onto the DC magnetic bias (generated by an electromagnet) [12-13]. All measurements were performed at room temperature.

\section{Results and discussion}

When a magnetic field applies to the capacitor system, the length of the magnetostrictive Terfenol-D changes, the distance between the parallel plates $(d)$ is changing accordingly. Therefore the capacitance of the capacitor changes. Figure. 2 shows the capacitance $(C)$ as functions of DC bias field $H$ at the signal magnetic field (about $0.45 \mathrm{Oe}$ ) frequency of $50 \mathrm{kHz}$. Figure 2 shows that the capacitance significantly affected by the DC magnetic field. $C$ increases with increasing $H$. Some previous studies indicate that the magnetostriction of Terfenol-D is affected by the DC magnetic field [14]. When $H$ is applied to the Terfenol-D, its length will increase with increasing $H$, and the distance between the parallel plates $d$ decreases. Therefore, the increase of $C$ with increasing $H$ is obtained based on equation below

$$
C=\varepsilon A / d
$$

Where $\varepsilon$ is the dielectric constant of the capacitor, $A$ is the plate area.

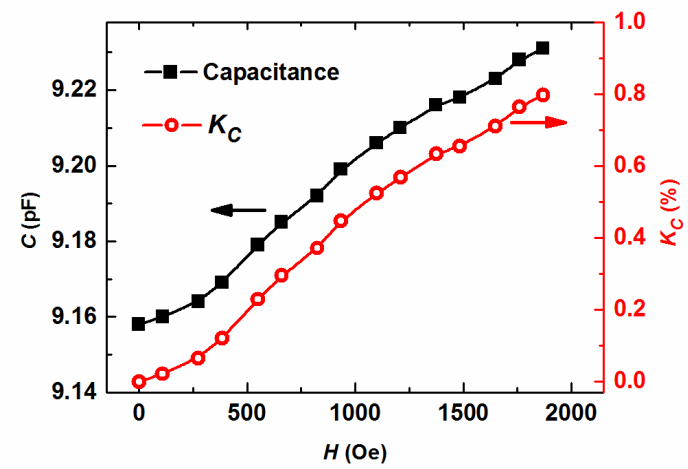

Figure 2. The capacitance $(C)$ and the magnetocapacitance effect of the capacitance $(M C)$ as functions of DC bias magnetic field $(H)$ at the frequency of $50 \mathrm{kHz}$ for the system.

Based on the strong field dependence of $C$, it is worthwhile to investigate the magnetocapacitance effect $(M C)$ with varying $H$. $M C$ can be expressed as

$$
M C=100 \% \times(\alpha(\mathrm{H})-\alpha(0)) / \alpha(0)
$$

Where $C(H)$ and $C(0)$ are the capacitance under an applied DC magnetic field and zero field at the frequency of $50 \mathrm{kHz}$, respectively. $M C$ increases with increasing $H$ as shown in Figure 2. both the $C$ and the $M C$ with $H$ show the same changing trend as magnetostriction of Terfenol-D does[14]. The result coincide with the equation (1). The maximum value of $K_{M}$ is about $0.8 \%$ at $H=1760$ Oe. This means that hat this system can be used in magnetic detectors and sensors.

In the parallel plate capacitor system, the electret film maintains a fixed charge. Therefore, a voltage across the parallel plate is obtained. As a magnetic field is applied to the Terfenol-D rod, it moves back and forth, changing the distance between the two plates of the capacitor system, which, in turn, creates an induced voltage difference [12]. When an AC signal magnetic field is superimposed on the DC magnetic field $H$, an AC signal will be induced and can be measured by an lock-inamplifier. The ME effect coefficient can be expressed as:

$$
\alpha_{M}=\delta U / \delta H
$$

Where $\delta H$ is the AC signal magnetic field, $\delta U$ is the induced voltage.

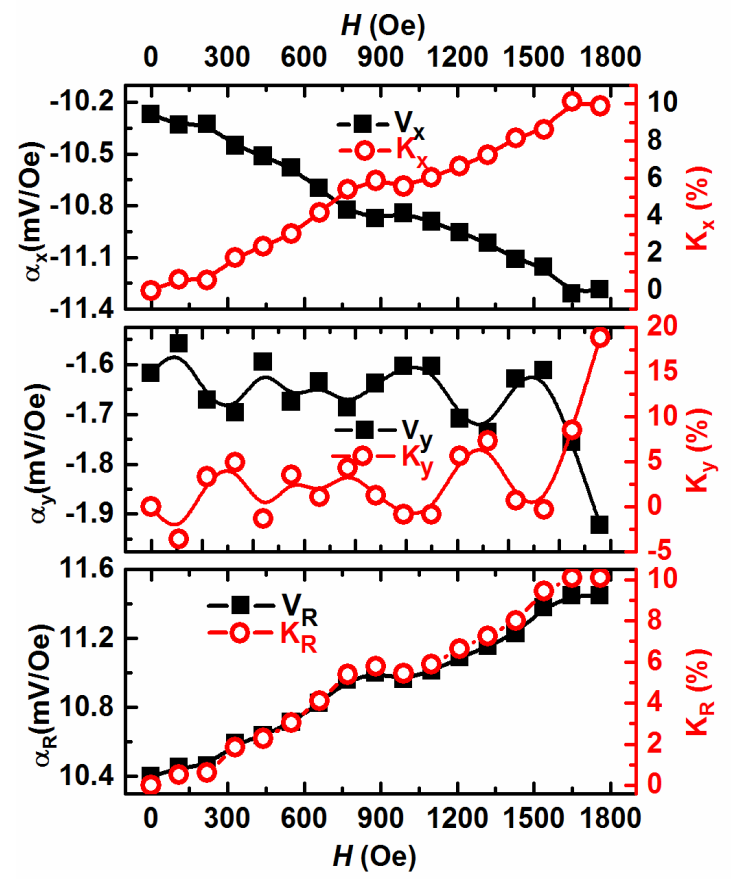

Figure 3. The real part, image part and effective values of the ME effect coefficient $\left(\alpha_{x}, \alpha_{y}\right.$ and $\left.\alpha_{R}\right)$ and their tunabilities $\left(K_{x}\right.$, $K_{y}$ and $K_{R}$ ) as functions of DC bias magnetic field $(H)$ at the frequencie of $50 \mathrm{kHz}$ for the system.

Figure 3 shows that the real part, image part and effective values of the ME effect coefficient $\left(\alpha_{x}, \alpha_{y}\right.$ and $\left.\alpha_{R}\right)$ as functions of DC bias magnetic field $(H)$ at the frequency of $50 \mathrm{kHz}$ for the system. The result shows that both $\alpha_{x}$ and $\alpha_{R}$ are significantly affected by the DC magnetic field. $\alpha_{x}$ decreases almost linearly with increasing $H$, while $\alpha_{R}$ increases almost linearly with increasing $H$. However, the changes of $\alpha_{y}$ with $H$ is not evident. $\alpha_{R}$ can be expressed as

$$
\alpha_{R}^{2}=\alpha_{x}^{2}+\alpha_{y}^{2}
$$

So the positive value of $\alpha_{R}$ can be observed as shown in Figure 3. 
Based on the strong $H$-dependence of the ME effect coefficient, it is worthwhile to investigate its tunability $(K)$ with varying $H$. $K$ can be expressed as

$$
K=100 \% \times|(\alpha(H)-\alpha(0)) / \alpha(0)|
$$

where $\alpha(H)$ and $\alpha(0)$ are the ME effect coefficient under an applied DC magnetic field and zero field, respectively. The tunabilities of $\alpha_{x}$ and $\alpha_{R}\left(K_{x}, K_{R}\right)$ increase almost linearly with increasing $H$ as shown in Figure 3. The highest value of $K_{x}, K_{R}$ are more than $10 \%$ at $H=1760 \mathrm{Oe}$, which indicates that this capacitor system may be used in magnetic detectors and sensors.

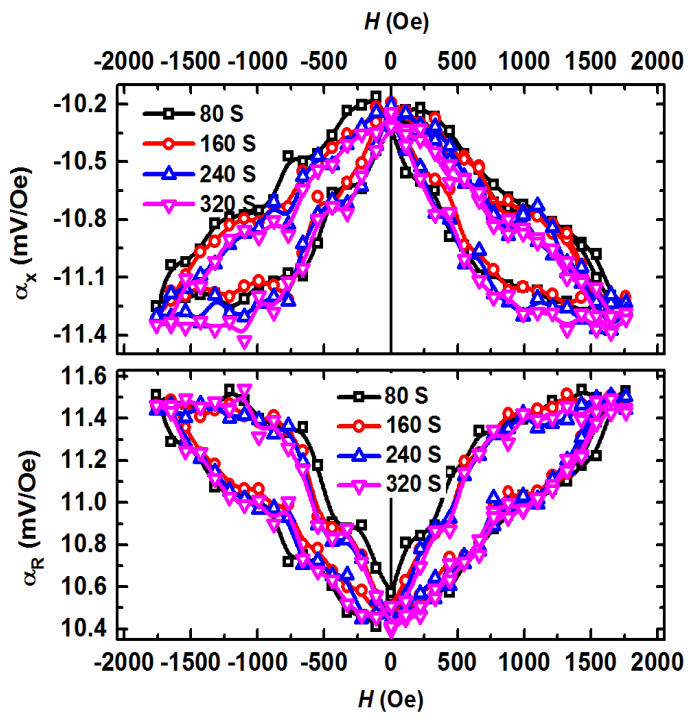

Figure 4. Test period dependence of the real part and effective values of the ME hysteresis effect coefficient $\left(\alpha_{x}, \alpha_{R}\right)$ loops at the applied signal field frequency of $50 \mathrm{kHz}$.

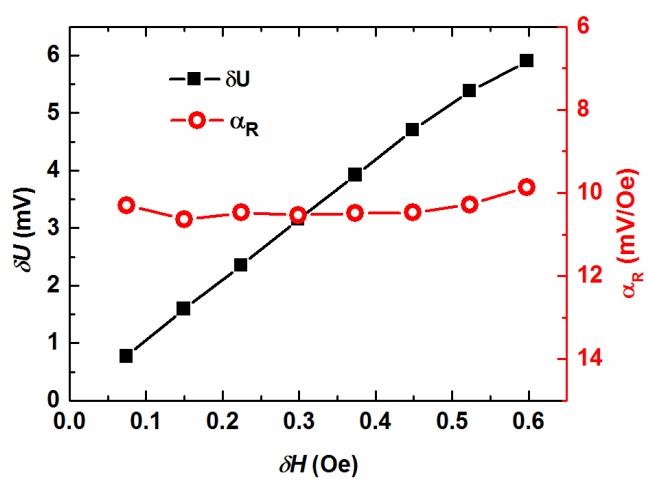

Figure 5. Induced AC voltage of effective values $(\delta U)$ and the effective values of the ME effect coefficient $\left(\alpha_{R}\right)$ as a function of applied AC signal magnetic field $(\delta H)$ at the frequency of 50 $\mathrm{kHz}$.

Figure 4 shows the test period dependence of the real part and effective values of the ME hysteresis effect coefficient $\left(\alpha_{x}, \alpha_{R}\right)$ loops at the applied signal field frequency of $50 \mathrm{kHz}$. Both $\alpha_{x}$ and $\alpha_{R}$ vs. $H$ have been measured by varying $H$ from zero to a positive maximum (1760 Oe), then from the positive maximum to a negative maximum (-1760 Oe), and finally, from the negative maximum to a positive maximum. This is a test period (T). As $H$ increases from zero to a positive maximum, $\alpha_{x}$ decreases to a minimum value, while $\alpha_{R}$ increases to a maximum value. As $H$ decreases from positive maximum to zero, both $\alpha_{x}$ and $\alpha_{R}$ do not reach their previous values, thereby exhibiting hysteresis. As $H$ is reversed from zero to the negative maximum, the same behavior is observed. The hysteresis behavior indicates that the ME effect of the system needs time to respond to the varying $H$. To see the ME effect with test period $(T)$, we measured the ME effect of the system with $T$ at $80,160,240$ and $320 \mathrm{~s}$, as shown in Figure 4. The result shows that both the real part and effective values of the ME hysteresis loops are almost unchanged with $T$, which is much different from that of the ME composites [13].

To explore the correlation of the effective value of the induced $\mathrm{AC}$ voltage $(\delta U)$ with the applied AC magnetic signal $(\delta H)$, the values of $\delta U$ with the applied $\delta H$ at frequency of $50 \mathrm{kHz}$ was measured, as shown in Figure 5 . The result indicate that with the increase of $\delta H, \delta U$ increases almost linearly, which means that this system can be used in AC magnetic detectors and sensors. The effective value of the ME effect coefficient $\left(\alpha_{R}\right)$ of 50 $\mathrm{kHz}$ is also obtained by $\delta U / \delta H$ as shown in Figure $5 . \alpha_{\mathrm{R}}$ is almost unchanged with the increase of $\delta U$. However, as $\delta U$ is too big, the $\alpha_{R}$ is slightly bigger than the others. The reason for the phenomenon is that the magnetostriction of Terfenol-D almost increase linearly with the increasing magnetic field at a low value range [14]. The signal magnetic field $(\delta H)$ is very small and less than 0.5 Oe, so the changing distance between the two plates of the capacitor $(\delta d)$ increases linearly with increasing $\delta H$. Accordingly, $\delta U$ increases linearly with increasing signal magnetic field as shown in Figure 5.This means that the AC magnetic signal should not be big in the ME effect measurement for this kind of system. Inaddition, this means that this capacitor system can be used in AC magnetic detectors and sensors.

\section{Conclusions}

In summary, we prepared a capacitor system composed of a Terfenol-D rod and an electret film, and studied the ME effect coefficient using dynamic measurement. A significant $\mathrm{ME}$ effect with high tunability at the frequency of $50 \mathrm{kHz}$ was observed. The results indicate that both the real part and effective values of the ME effect coefficient are strongly dependent on the bias magnetic field. The bias-field-dependent ME hysteresis of the system is almost unchanged with the test periods. The induced effective value of AC voltage increases almost linearly with the increasing AC magnetic signal. The results indicate that this type of capacitor system has potential practical uses in energy converter, magnetic detection and sensors.

\section{Acknowledgment}

This work is supported by the National Natural Science Foundation of China (NSFC No. 11547048). 


\section{References}

1. Fiebig M, J. Phys. D: Appl. Phys. 38, R123 (2005)

2. Fusil S, Garcia V, Barthélémy A and Bibes M, Annu.

Rev. Mater. Res. 44,91 (2014)

3. Gonçalves R, Larrea A, Zheng T, Higgins M J,

Sebastian V, Lanceros-Mendez S and Martins P, Eur.

Polym. J. 84, 685(2016)

4. Palneedi H, Maurya D, Kim G Y, Priya S, Kang S J L, Kim K H, Choi S Y and Ryu J, Appl. Phys. Lett. 107, 012904 (2015)

5. Tian Y, Shen S, Cong J, Yan L, Wang S and Sun Y, J. Am. Chem. Soc. 138, 782 (2016)

6. Chen A, Zhao Y, Li P, Zhang X, Peng R, Huang H, Zou L, Zheng X, Zhang S, Miao P, Lu Y, Cai J, Nan CW, Adv. Mater. 28, 363 (2016)

7. Ummer R P, Raneesh B, Thevenot C, Rouxel D,

Thomas S and Kalarikkal N, Rsc Adv. 6, 28069 (2016)

8. McCarty L S, Whitesides G M, Angew, Chem. Int. Edit. 47, 2188 (2008)

9. Chen G, Li Y, Xiao H and Zhu X, J. Mater. Chem. A

$\mathbf{8 , 5}(2017)$

10. Chen G, Xu Y, Chen X, XIao H, 25, 797(2018)

11. Shin K, Kim J, Lee H, Seo D and Moon W, J. Acoust. Soc. Am. 140, 3033 (2016)

12. Zhou Y, Chen G, Sun Y, Sun Y., Zhou H, Cao D, Xu L, Jiao Z, Sensor. Actuat. A-Phys. 270,46( 2018)

13. Zhou Y, Zhou H M, Ye Y X and Jiao Z W, Appl. Phys. A 122,142 (2016)

14. Zheng X J, Liu X E, J. Appl. Phys. 97, 053901 (2005) 